\title{
Synchronization of surface electromyography and 3D electromagnetic articulography applied to study the biomechanics of the mandible: proof of concept.
}

Florencia Lezcano

Universidad de La Frontera

Fernando Jose Dias

Universidad de La Frontera

Constanza Farfán

Universidad Internacional de Andalucia - Campus de Huelva Sede Santa Maria de La Rabida

María Cristina Manzanares Céspedes

Universitat de Barcelona

Ramon Fuentes ( $\square$ ramon.fuentes@ufrontera.cl)

Universidad de La Frontera https://orcid.org/0000-0002-5895-024X

Research article

Keywords: Electromagnetic articulography, surface electromyography, biomechanics, mandible, chewing

Posted Date: November 11th, 2019

DOl: https://doi.org/10.21203/rs.2.16961/v1

License: (c) (1) This work is licensed under a Creative Commons Attribution 4.0 International License.

Read Full License 


\section{Abstract}

Background. The aim of this study was to propose and establish the proof of concept of an approach to synchronize 3D Electromagnetic Articulography (3D-EMA) with Surface Electromyography (SEMG) systems based on the standard components of these equipment.

Methods. Initially it was planned and obtained the required equipment with the appropriate characteristics required, and them it was created the proper conditions to register the synchronized signals provided by both systems. Thus, we selected a SEMG with a switch module incorporated to be able to achieve synchronization of signals. After the system setup was stablished, chewing tasks were recorded on a healthy volunteer, collecting a proof-of-concept database. The variability among recordings of the database were analyzed in order to detect possible interferences.

Results. The analysis of the chewing task recordings obtained with the synchronized 3D EMA and SEMG signals in the present study did not reveal significant distortions, all values were within the informed by the manufacturers of both systems. The method presented the advantage of using only components that are already included with the equipment employed.

Conclusion. The method of analysis described in this paper is an effective tool that facilitates the investigation of mandibular movements synchronized in two domains: articulatory movements and electromyographic activity. Thus, it is promising to be applied in different clinical situations to improve the analysis of the complexity of masticatory activity, in addition being able to generate new insights on this topic.

\section{Background}

For a complete understanding of the kinesiology of the mandible, it is necessary to record muscle activity and movement pattern simultaneously as a strategy to relate the phases of chewing cycles to muscular contractions [1]. Neuromuscular analyses involving surface electromyography (SEMG) of the masticatory muscles and kinematics of the mandible have been widely employed in clinical practice and research. These techniques allow quantification of disharmony in pathological conditions as well as the outcome of given therapies [2]. Some studies involved simultaneous recordings of both kind of signals [1,3-16] but only a few achieve synchronization $[1,3-5,15]$. To synchronize signals from different acquisition systems is a challenging task and the methodology employed by researchers is not always clearly reported.

Current advances in technology have allowed dentists precise methods to evaluate mandibular movements (MM) and muscle activity. Surface EMG is a non-invasive technique that provides information on muscle properties through electrodes located over the skin. The surface EMG is the sum of the electrical contributions of the active motor units and reflects both the muscle membrane properties and the central control strategies [17]. Many researchers apply surface EMG recordings in both basic research and clinical studies [18]. In controlled experimental conditions, surface EMG has been shown to 
be a powerful tool to study the jaw elevator muscles physiology, muscle impairments associated to different temporomandibular disorders (TMD), to detect muscle hyper-and hypo-activity, muscle imbalance, rest position and fatigue [18].

Electromagnetic articulography (EMA) is a non-invasive technique which can be employed to analyze kinematics of the mandible. It is based on the inductive measurement of distances, originally introduced to track the movement of articulators such as tongue and lips during speech production [19]. EMA systems generate an alternating magnetic field of controlled frequency in the measurement area by the implementation of transmitter coils [19-21]. The magnetic field induces an alternating electric current in small receiver coils also called sensors [19]. The induced current is analyzed to calculate the spatial position of the sensors [19]. Fuentes et al. employed the AG501 articulograph to assess cinematic characteristics of masticatory cycles in healthy participants with normal occlusion tracking the position of an EMA sensor placed in the interincisal midline of the mandible [22-24]. Hoke et al. employed the Wave articulograph to analyze the micro-movement of dental prosthesis during mastication [25]. Compared with other methods employed to track MM, EMA systems allow more natural movements because it does not restrict the movement of body including the head, and it also achieves high spatial precision (up to $0.3 \mathrm{~mm}$ ) and temporal precision (sample frequency of up to $1 \mathrm{KHz}$ ) $[22-23,26]$.

Fusing electromagnetic articulography with surface electromyography provides new opportunities to analyze the biomechanics of the mandible. Our goal is to propose and establish the proof-of-concept of an approach to synchronize a 3D electromagnetic articulograph with a surface electromyograph based on the standard components of the equipment.

\section{Methods}

At first, it was necessary to plan and gather the required equipment. The next step was to create the necessary conditions to register and synchronize the signals provided by the different systems. For this purpose, we selected a surface electromyograph with a switch module incorporated, which is usually employed to synchronize the EMGs signal with mechanical events. After the system setup was ready, a proof-of-concept database was collected for further analysis.

\section{Equipment and system setup}

A 3D electromagnetic articulograph (AG501, Carstens Medizinelektronik, Germany) was selected to register MM. This equipment is classified as Low Power Communication Device Transmitter by the Federal Communications Commission of the United States (FCC ID 2AHFP-AG501). A surface electromyograph (SEMG VIII, ArtOficio, Chile) was selected to register the electromyographic activity of the Temporal (anterior portion; right and left) and Masseter (right and left) muscles. An essential accessory to achieve the proposed synchronization is the EMGs "switch". When pressed, this accessory generates an electrical event on the SEMG signal and at the same time, an acoustic signal that can be easily recorded using the EMA microphone. The audio recording is synchronized with the articulographic 
recordings. Therefore, when synchronizing SEMG and audio signals, all articulographic recordings will be synchronized too.

The main characteristics of the equipment employed are summarized in Table 1. The setup was arranged as shown in Figure 1. The EMA microphone and the EMG switch should be close from each other.

\section{Recording protocol}

In order to conduct a simultaneous recording for posterior synchronization, the next sequence of steps should be followed:

- The subject under study should be located and connected as shown in Figure 1.

- To register an experimental task, both systems should start recording before conducting the task.

- The EMG switch is pressed to generate a visible event in the signals of both systems.

- The experimental task is carried out.

- Both systems stop recording.

- EMA and EMG data are saved as binary files or text files.

- Steps 1 to 5 are repeated until finishing all experimental tasks.

- All resulting files are organized within a folder containing all the recordings made within the whole session.

\section{Off-line signal processing and synchronization}

The recordings are processed off-line in order to achieve synchronization. The peeks in the audio signal and the squared signal registered by the SEMG, both events generated by the same switch, are employed to synchronize the signals of both systems as shown in Figure 2. The switch was pressed three times at the beginning of the simultaneous recording. Three square pulses of $5 \mathrm{~V}$ of amplitude can be observed in the SEMG signal and six peeks can be observed in the audio recording. Both signals are synchronized when the peeks coincide with the flanks of the square pulses.

\section{Proof-of-concept database}

To prove the viability of the proposed methodology, the following recordings were made in a healthy volunteer (Ethics Committee Approval number 125/18):

- Articulographic recording the chewing of $3.7 \mathrm{~g}$ of peanuts placing 3 active sensors on the jaw: in the interincisal midline, between first right molar and premolar and between left molar and premolar.

- Electromyographic recording of the chewing of $3.7 \mathrm{~g}$ of peanuts. 
- Simultaneous electromyographic and articulographic recording of the chewing of $3.7 \mathrm{~g}$ of peanuts.

Each chewing task was performed two times. First, chewing to the right side and then to the left side. These recordings were organized and saved, generating a proof-of-concept database.

\section{Accuracy analysis}

The Proof-of-concept database (EMA and EMG data) was processed to analyze the differences between simultaneous and individual recordings employing Matlab (R2019a, MathWorks, USA). The following parameters were calculated:

- Standard deviation of instantaneous measurements of Euclidean distance between pairs of sensors for each articulographic recording

- Average of the EMGrms (40 ms window) signal of each electromyographic recording

The variation of both parameters between the different registration modalities (simultaneous and individual) were analyzed.

\section{Results}

Regarding the analysis of the articulographic recordings of chewing, the standard deviation of the distance measurements between the sensors was $0.2 \mathrm{~mm}$ for the simultaneous registration of EMA with EMG and $0.3 \mathrm{~mm}$ for the individual registration of EMA. The values were within the limits indicated by the manufacturer. Figure 3 shows the trajectory of the mid-incisal point registered with EMA with and without connecting the SEMG device to register the muscle activity.

A difference between $1 \%$ and $10 \%$ was found between the means of the EMGrms (Figure 4) associated to the simultaneous EMG records with EMA and individual EMG respectably (Table 2).

Finally, the achieved synchronization of EMGrms and MM signals is shown in Figure 5. Only the SEMG recording of chewing from the right Masseter Muscle is shown. Scales were adjusted to achieve a better representation of the relation of both signals.

\section{Discussion}

The present study proposed and established a Proof-of-concept for the synchronization of two electromedical sytems, 3D electromagnetic articulography and surface electromyography, explaining it in detail so that it can be replicated by other research groups. The implementation of the synchronization and further analysis aims to improve understanding of the complexity of mandibular functional movements which may be applied to study different clinical situations. 
The study of functional movements through analysis of kinematics and muscle activity is widely approached in various disciplines [27], including dentistry [1-3,5,7-9,11,13-14,16,28], which reveals the importance of analyzing these complex movements by various methods that complement each other.

Simultaneous measurements of different biomechanical variables of mandibular movements have been carried out for many years. Bigland measured the speed of contraction, the EMG activity, and the tension applied controlled by putting different weights [29]. More recent studies have also analyzed mandibular movements with simultaneous recordings of electromyographic activity and jaw movements in dentate individuals [8] and patients with maxillary and mandibular complete dentures [13] without synchronizing both recordings. These studies, even with clinical value, have its limitations due to the lack of synchronization of their recordings or lack of more detailed explanation of the methodology employed. Thus, limiting the extrapolation of their results in the clinical understanding of mandibular movements that reveal the need for a more complete analysis.

Other authors have developed studies demonstrating the concern to synchronize the signals recorded by different electromedical equipment related to mandibular movements with the electromyographic activity of the muscles of the stomatognathic system [6-7,9-11,14], however, these studies did not provide enough information regarding the methods to be replicated.

Some studies have shown the synchronization between electromedical equipment applied to different analyses of the stomatognathic system, using arrangements or equipment specially developed for this purpose. Widmalm et al. designed and built a special device to combine recordings of vibration and EMG activity [30]. Nakazawa et al. performed a master-slave arrangement for palatal pressure, mandibular movement, and EMG recordings with $1 \mathrm{~ms}$ delay in the synchronization [31]. Kerstein used a specific synchronization module for EMG signal analysis and occlusal force distribution analyzed using the TSCAN system [32-33]. And Grigoriadis et al. synchronized EMG records of masseter muscle and mandibular movements using 2D magnetic sensors using a commercial system [16].

Other studies performed the synchronization of the recordings by generating an independent and identifiable event in the signals acquired by different systems. Ahlgren used a light signal to synchronize EMG and cinematography recordings [1]. A pulse generator was used to synchronize EMG activity and mandibular movements by videofluorography and cineradiography [3-5]. Freitas et al. synchronized the recordings of mandibular movements obtained by a Kinect sensor and EMG activity through a computational custom made system [15].

Synchronization through the generation of a specific event was the method chosen by our research group to synchronize the EMG recordings of masticatory muscles and mandibular movements. In our case, we used a "switch" device that comes with EMG equipment and a sound recorder that comes with AG501 3DEMA equipment. Activation of this switch generated an independent and distinguishable event in both registers, which in the case of EMG was recorded as a square electrical signal and in the EMA a peek in the acoustic signal, which was used to synchronize both recordings. 
Our recording method is a novelty in the scientific literature, and has the advantage of the high accuracy of the movement-recording method demonstrated in previous articles from our research group [22-24]. In addition, this synchronization was performed using only the components that already accompany both equipment selected, a fact that besides facilitating its realization and replication, does not add costs to its realization.

In general, our results obtained during synchronized recordings showed no distortion/alteration outside the limit values informed by the manufacturers. Even though both instruments are very sensitive to electromagnetism, the EMA AG501 and the EMGs proposed in this methodology do not generate interferences between them during the simultaneous recording. Probably because the bandwidths with which each system works do not overlap.

\section{Conclusions}

The present study was able to establish a detailed proof of concept of the synchronization of chewing muscle EMG activity related to mandibular movements, recorded by 3D-EMA, without generating significant distortion (within the values informed by the manufacturers), with the advantage of using components that are included with the equipment employed. The measurement system described in this paper is an effective tool that facilitates simultaneous investigation of mandibular movements in two domains: articulatory movements and electromyographic activity of muscles. Thus, it is promising to be applied in different clinical situations to improve the analysis of the complexity of masticatory activity, thus generating new insights on this topic.

\section{Declarations}

LIST OF ABBREVIATIONS

Not applicable.

\section{Ethics approval and consent to participate}

The subject under study has participated voluntarily, signing informed consent. Ethics Committee Approval number 125/18, "Comité Ético Científico", University of La Frontera, Chile.

\section{Consent for publication}

The participant has given consent for publication.

\section{Availability of data and materials}


The datasets used and/or analysed during the current study are available from the corresponding author on reasonable request.

\section{Competing interests}

The authors declare that they have no competing interests.

\section{Funding}

Ramón Fuentes and his research group have received financial support from the Research Office, Universidad de La Frontera. This work is part of project DFP18-0037.

\section{Authors' contributions}

FL, RF and MCM conceived and designed research. FL and CF conductedexperiments. FL contributed new analytical tools. FL and CF analyzed data. FL and FDwrote the manuscript. All authors read and approved the manuscript.

\section{Acknowledgements}

Not applicable.

\section{References}

1. Ahlgren J. Kinesiology of the mandible an EMG study. Acta Odontol Scand. 1967;25(6):593-612.

2. Campillo B, Martín C, Palma JC, Fuentes AD, Alarcón JA. Electromyographic activity of the jaw muscles and mandibular kinematics in young adults with theoretically ideal dental occlusion: Reference values. Med Oral Patol Oral Cir Bucal. 2017;22(3):e383.

3. Pancherz $\mathrm{H}$, Winnberg A, Westesson PL. Masticatory muscle activity and hyoid bone behavior during cyclic jaw movements in man: a synchronized electromyographic and videofluorographic study. Am J Orthodont. 1986;89(2):122-131.

4. Hylander $\mathrm{WL}$, Johnson KR, Crompton AW. Loading patterns and jaw movements during mastication in macaca fascicularis: a bone-strain, electromyographic, and cineradiographic analysis. Am J Phys Anthropol. 1987;72(3):287-314.

5. Winnberg A, Pancherz H, Westesson PL. Head posture and hyo-mandibular function in man: a synchronized electromyographic and video fluorographic study of the open-close-clench cycle. Am J Orthod Dentofac Orthop. 1988;94(5):393-404. 
6. Gerstner GE, Goldberg LJ. An analysis of mandibular movement trajectories and masticatory muscle EMG activity during drinking in the guinea pig. Brain Res. 1989;479(1):6-15.

7. Ottenhoff FA, Van Der Bilt A, Van Der Glas HW, Bosman F. Control of elevator muscle activity during simulated chewing with varying food resistance in humans. J Neurophysiol. 1992;68(3):933-944.

8. Van Eijden TMGJ, Blanksma NG, Brugman P. Amplitude and timing of EMG activity in the human masseter muscle during selected motor tasks. J Dent Res. 1993;72(3):599-606.

9. Hiraba K, Hibino K, Hiranuma K, Negoro T. EMG activities of two heads of the human lateral pterygoid muscle in relation to mandibular condyle movement and biting force. $J$ Neurophys. 2000;83(4):2120-2137.

10. Igarashi N, Yamamura K, Yamada Y, Kohno S. Head movements and neck muscle activities associated with the jaw movement during mastication in the rabbit authors. Brain Res. 2000;871(1):151-155.

11. Van Der Bilt A, Weijnen FG, Bosman F, Van Der Glas HW, Kuks JB. Controlled study of EMG activity of the jaw closers and openers during mastication in patients with myasthenia gravis. Eur J Oral Sci. 2001;109(3):160-164.

12. Naganuma $K$, Inoue $M$, Yamamura $K$, Hanada $K$, Yamada $Y$. Tongue and jaw muscle activities during chewing and swallowing in freely behaving rabbits. Brain Res. 2001;915(2):185-194.

13. Piancino MG, Farina D, Talpone F, Castroflorio T, Gassino G, Margarino V, Bracco P. Surface EMG of jaw-elevator muscles and chewing pattern in complete denture wearers. J Oral Rehab. 2005;32(12):863-870.

14. Piancino MG, Bracco P, Vallelonga T, Merlo A, Farina D. Effect of bolus hardness on the chewing pattern and activation of masticatory muscles in subjects with normal dental occlusion. $J$ Electromyogr and Kinesiol. 2008;18(6):931-937.

15. Freitas J, Teixeira A, Dias MS. Multimodal silent speech interface based on video, depth, surface electromyography and ultrasonic doppler: data collection and first recognition results. In: Speech Production in Automatic Speech Recognition. International Speech Communication Association. 2013. http://www.isca-speech.org/archive/spasr_2013. Accessed 10 Sep 2018.

16. Grigoriadis A, Johansson RS, Trulsson M (2014) Temporal profile and amplitude of human masseter muscle activity is adapted to food properties during individual chewing cycles. $J$ Oral Rehab. 2014;41(5):367-373.

17. Basmajian JV. Electromyography: its structural and neural basis. Int Rev Cytology. 1967;21:129-140.

18. Castroflorio T, Bracco P, Farina D. Surface electromyography in the assessment of jaw elevator muscles. J Oral Rehab. 2008;35(8):638-645.

19. Hixon TX. An electromagnetic method for transducing jaw movements during speech. J Acoust Soc Am. 1971;49(2B):603-606.

20. Schönle PW, Gräbe K, Wenig P, Höhne J, Schrader J, Conrad B. Electromagnetic articulography: use of alternating magnetic fields for tracking movements of multiple points inside and outside the vocal tract. Brain Lang. 1987;31(1):26-35. 
21. Kaburagi T, Wakamiya $K$, Honda M. Three-dimensional electromagnetic articulography: a measurement principle. J Acoust Soc Am. 2005;118(1):428-443.

22. Fuentes $R$, Arias A, Saravia D, Lezcano MF. An innovative method to analyse the range of border mandibular movements using 3D electromagnetic articulography (AG501) and MATLAB. Biomed Res. 2017;28(9):4239-4247.

23. Fuentes R, Arias A, Lezcano MF, Saravia D, Kuramochi G, Dias FJ. Systematic standardized and individualized assessment of masticatory cycles using electromagnetic 3D articulography and computer scripts. Biomed Res Int. 2017;2017:1-9.

24. Fuentes R, Arias A, Lezcano MF, Saravia D, Kuramochi G, Navarro P, Dias FJ (2018) A new tridimensional insight on geometric and kinematic characteristics of masticatory cycles in participants with normal occlusion. Biomed Res Int. 2018;2018:1-9.

25. Hoke P, Tiede M, Grender J, Klukowska M, Peters J, Carr G. Using electromagnetic articulography to measure denture micromovement during chewing with and without denture adhesive. J Prosthodont. 2019;28(1):e252-e258.

26. Fuentes R, Navarro P, Curiqueo A, Ottone NE. Determination of mandibular border and functional movement protocols using an electromagnetic articulograph (EMA). Int J Clin Exp Med. 2015;8(11):19905.

27. De Luca CJ. The use of surface electromyography in biomechanics. J App Biomech. 1997;13(2):135-163.

28. Pruzansky S. The application of electromyography to dental research. J Am Dent Assoc. 1952;44(1): 49-68.

29. Bigland B, Lippold OCJ. The relation between force, velocity and integrated electrical activity in human muscles. J Physiol. 1954;123(1):214-224.

30. Widmalm SE, Hedegård B. An apparatus for the synchronous registration of EMG activity in jaw muscles and of vibrations in the masticatory system. J Oral Rehab. 1974;1(2):183-190.

31. Nakazawa F, Togashi M. Evaluation of food texture by mastication and palatal pressure, jaw movement and electromyography. Hydrocolloids. 2000;473-483.

32. Kerstein, RB. Combining technologies: a computerized occlusal analysis system synchronized with a computerized electromyography system. Cranio. 2004;22(2):96-109.

33. Kerstein RB. Reducing chronic masseter and temporalis muscular hyperactivity with computer-guided occlusal adjustments. Compend Contin Educ Dent. 2010;31(7):530-4.

\section{Tables}


Table 1 Equipment characteristics

\begin{tabular}{|l|l}
\hline \multicolumn{1}{|c|}{ Electromagnetic articulograph } & \multicolumn{1}{c}{ Surface electromyograph } \\
\hline AG 501, Carstens Medizinelektronik, Bovenden, & SEMG VIII, ArtOficio, Santiago, Chile \\
Germany & Sampling frequency 250 KHz \\
Sampling frequency 250 Hz and $1250 \mathrm{~Hz}$ & 8 channels \\
16 channels & BW from 10 Hz to $500 \mathrm{~Hz}$ \\
Electromagnetic field frequencies from 7.5 to $13.75 \mathrm{KHz}$ & Bipolar sensors \\
Sensors version HQ220-L120-B & PC with dedicated software \\
Notebook with dedicated software & Switch Module to synchronize with mechanical \\
& event \\
\hline
\end{tabular}

Table 2. EMGrms mean

\begin{tabular}{lccc}
\hline & Without EMA & With EMA & Difference \\
\hline LT & $31,6 \mu \mathrm{V}$ & $31,3 \mu \mathrm{V}$ & $1 \%$ \\
RT & $19,6 \mu \mathrm{V}$ & $20,1 \mu \mathrm{V}$ & $3 \%$ \\
LM & $15,8 \mu \mathrm{V}$ & $15,3 \mu \mathrm{V}$ & $3 \%$ \\
RM & $10,2 \mu \mathrm{V}$ & $9,2 \mu \mathrm{V}$ & $10 \%$ \\
\hline
\end{tabular}

\section{Figures}




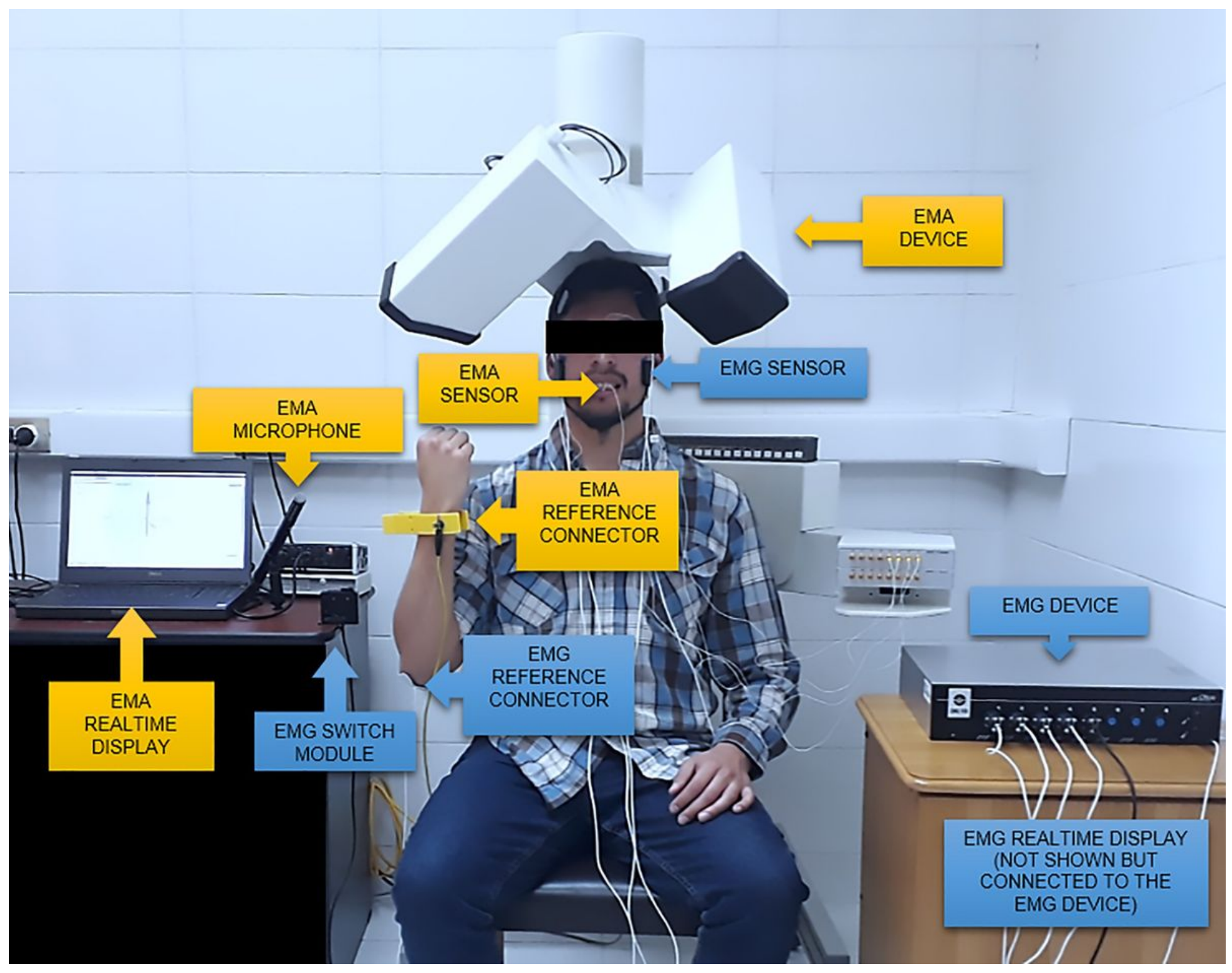

Figure 1

System setup. 

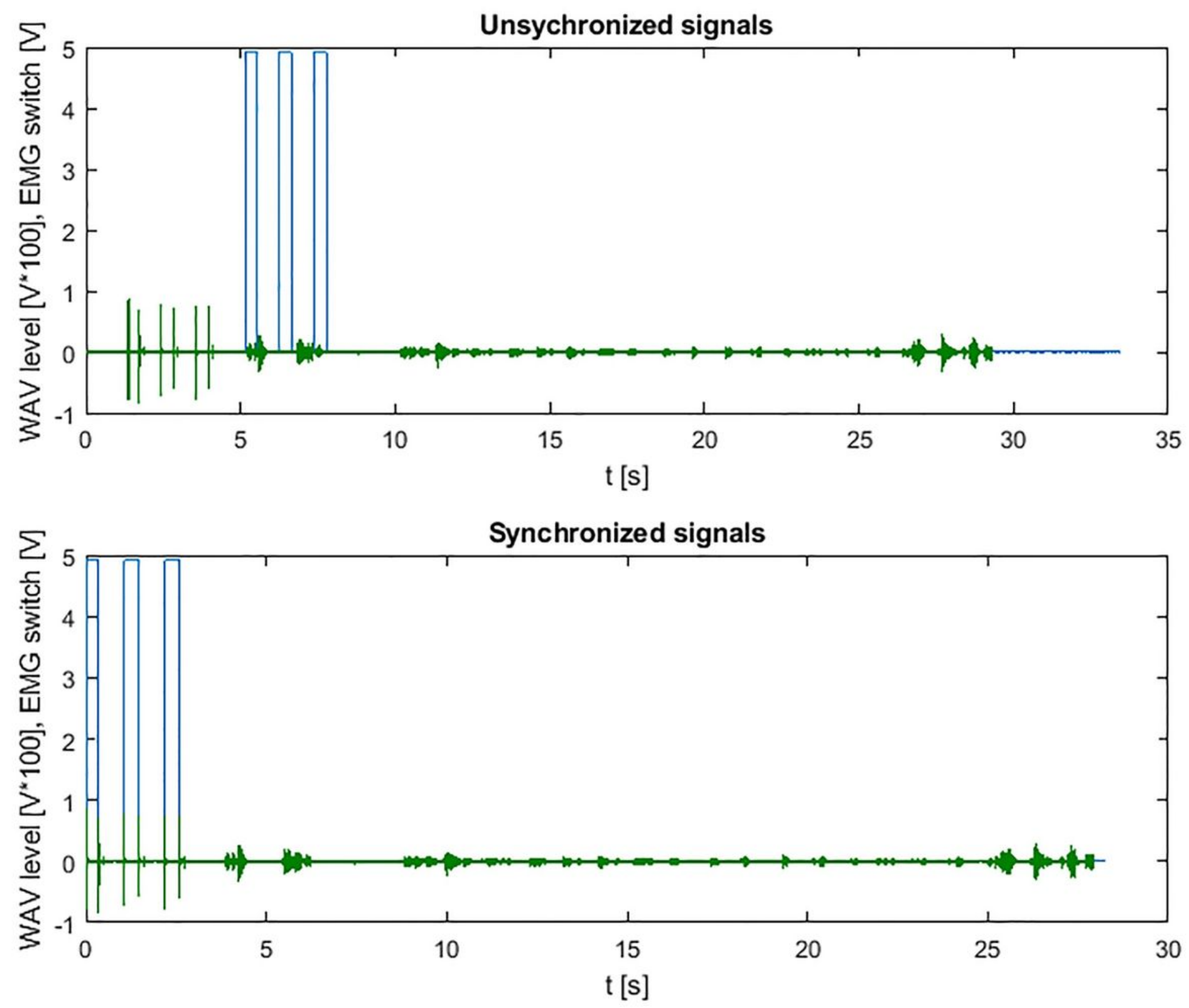

Figure 2

Synchronization of the switch signal of SEMG system (blue) and audio signal of EMA system (green)
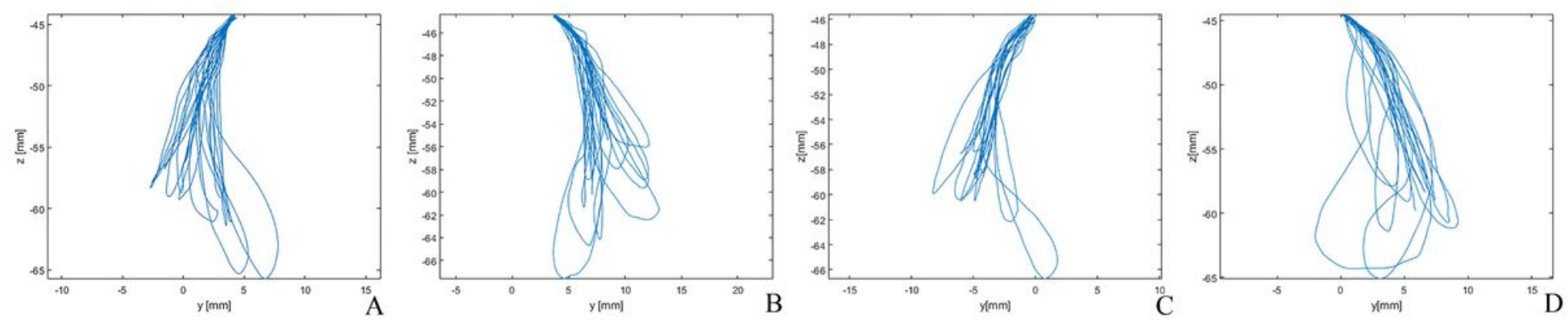

Figure 3 
EMA recordings: trajectories of the mid-incisal point. When combined with EMG, chewing to the right side (A) and to the left side (B). Without connecting the EMG device, chewing to the right side (C) and to the left side (D).
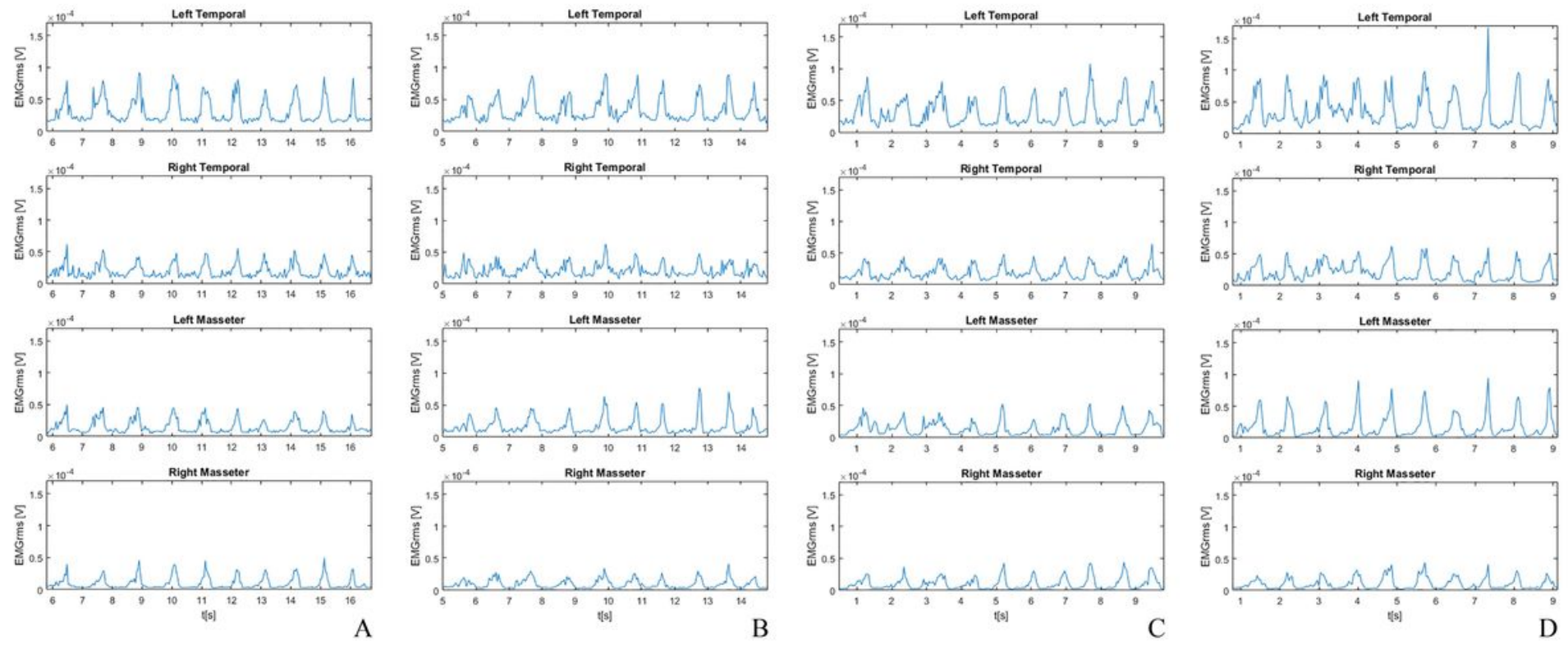

\section{Figure 4}

EMG recordings: EMGrms of Masseter and Temporalis (anterior portion). When combined with EMA, chewing to the right side (A) and to the left side (B). Without connecting EMA device, chewing to the right side (C) and to the left side (D). 


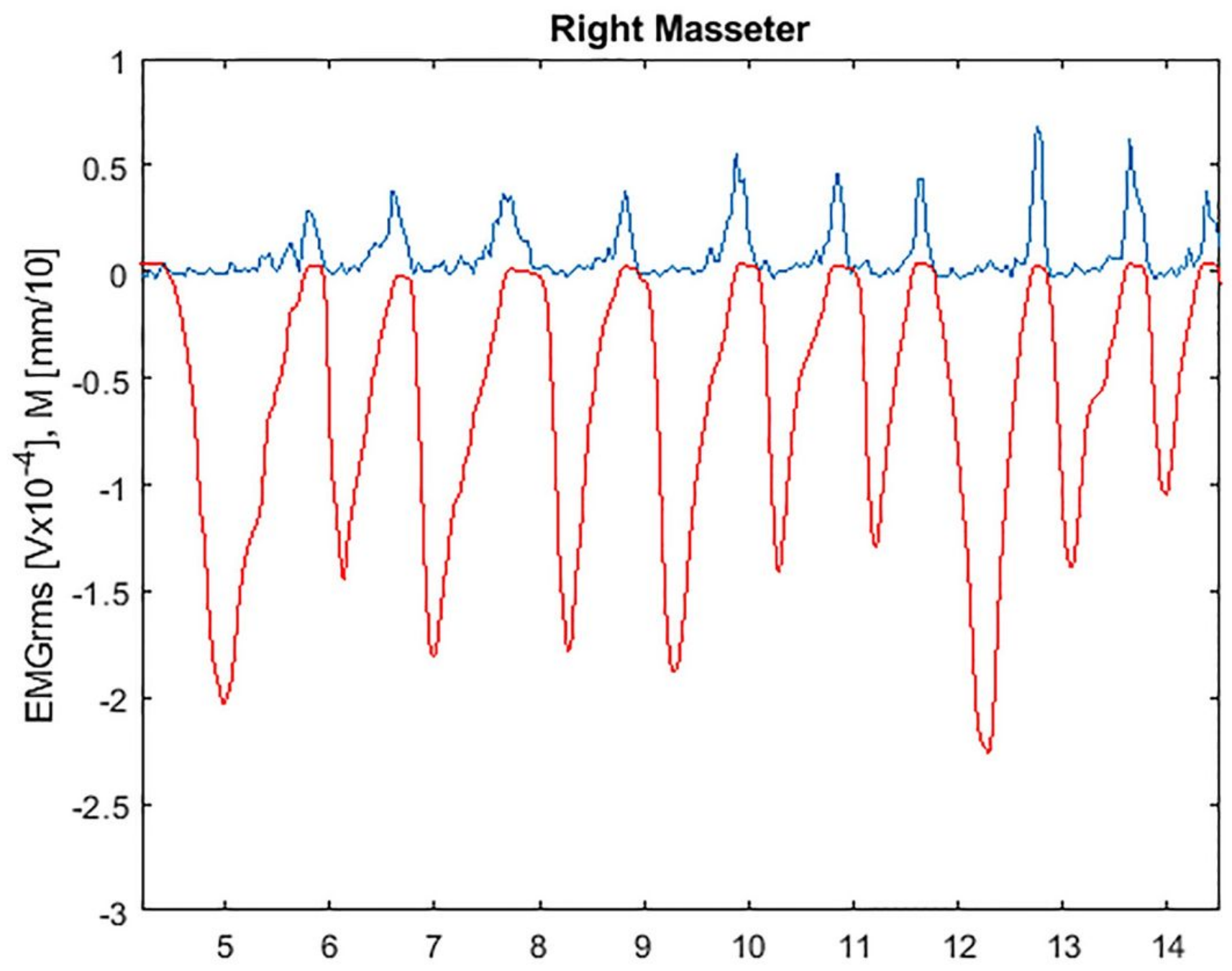

Figure 5

EMGrms signal from the right Masseter Muscle (blue) and MM (red) synchronized. 